\title{
Modified Time-Varying Damage Model with Caputo Fractional Derivative on the Creep Destruction Prediction of Rock Slope
}

\author{
Zhi-Xin Zhen (iD \\ School of Civil and Resource Engineering, University of Science and Technology Beijing, Beijing 100083, China \\ Correspondence should be addressed to Zhi-Xin Zhen; b20160029@xs.ustb.edu.cn
}

Received 25 October 2020; Revised 16 January 2021; Accepted 29 January 2021; Published 8 February 2021

Academic Editor: Toqeer Mahmood

Copyright (c) 2021 Zhi-Xin Zhen. This is an open access article distributed under the Creative Commons Attribution License, which permits unrestricted use, distribution, and reproduction in any medium, provided the original work is properly cited.

\begin{abstract}
Based on the consideration of the time effect and connecting with typical instances, the modified Caputo fractional time-varying damage model is proposed in this paper. The parameters of creep constitutive model of slope are determined by using the powerful mathematical software and the influence of the parameters of time varying viscus element and damage element, $\alpha$ and $\xi$, on strain are shown. As $\alpha$ increases, the strain rates of the first and second stages of creep attenuation and steady-state stages gradually decrease and the strain rate in the acceleration stage is reflected in $\xi$. The fitting curve is in good agreement with the experimental data, which proves that the damage model proposed in this paper is reasonable and accurate. It provides useful reference and guidance for further study of slope creep failure and practical applications.
\end{abstract}

\section{Introduction}

Creep is an important concept in rheological theory. Rheological mechanics stands for a key disciplinary area that studies the law of material stress-strain state with time, and its primary purpose is to study the mechanical properties of the basic purpose of real object and reflects the diversity and individuality of mechanics medium [1]. When it comes to the field of rheological mechanics, it pays more attention to the relationship between mechanical properties and material structure. By establishing the constitutive equation of the mechanical medium, the stress and deformation of the object under the action of external stress, especially the creep related to time factor, are studied [2].

There are two major types of rock slope: the natural slope formed naturally and the engineering slope of artificial excavation. Under the influence of various sudden factors, such as rainstorm and earthquake, the progressive failure occurs gradually and then landslides appear. The geological stress can cause different degrees of creep deformation occurred in the rock slope with the passage of time [3].

Engineering practice and research show that the failure and instability of rock slope engineering do not occur immediately after excavation in many cases, and the stress and deformation of rock slope develop and constantly adjust with time, and the adjustment process usually needs to continue for a long period. Creep of slope refers to the property that the deformation of rock mass of slope increases continuously with time under the action of dead weight stress and tectonic stress field dominated by horizontal stress. The deformation of slope is caused by many factors, such as geological action, underground water flow, temperature change, and vegetation action. In slope stability analysis, the slice of soil mass above failure surface plays a major role on factor of safety in which it has been considered as a circular slide line, general slide line, and inclined slices [4]. On the rock slope itself, the time-dependent deformation of it is mainly caused by the creep of the rock. Therefore, the close relationship between the creep characteristics of the rock and the creep characteristics of the slope should be paid special attention when studying the slope deformation. However, as far as the rock mass itself is concerned, the time-related deformation of slope is mainly caused by the creep of rock mass. Therefore, when studying the deformation of slope, special attention should be paid to the close relationship between the creep characteristics of rock and the creep characteristics of slope. 
Many engineering problems have been solved based on the damage theory, for example, constitutive equations of a nonlinear unsteady creep and a damage (the development of internal defects) were introduced by Elsoufiev [5]. A numerical model for long-term deformation and progressive failure of rock slope was presented by Liu et al. [6]. The dimensionless relationship model of the stress-strain of concrete on the basis of randomness was proposed in [7] by Liu et al. A variable-parameter restrictive shear creep model was established by Zhang to reflect the progressive-failure characteristics and describe the whole process of restrictive shear creep of the rock and the two failure mechanisms of the rock, which can provide the reference base for the prediction of the time of shear creep failure of the rock [8]. Riva et al. [9] proposed a damage-based, time-dependent $2 \mathrm{D}$ model of brittle creep, and the model investigates the mechanical role of deglaciation and damage-controlled fluid distribution in the development of alpine rockslides. A model of progressive damage through intact rock mass was developed by Lacroix and Amitrano [10]. Xue et al. [11] deduced two quantitative displacement criteria for slope deformation through the combination of a one-dimensional renormalization group model and the strain-softening constitutive model of geological materials. Umrao et al. [12] investigated the stability of the hill cut soil slopes and suggested possible stabilisation measures.

To solve the stability problem of rock slope, the creep model of rock slope should be analyzed and then the creep behavior and characteristics of rock slope are discussed. Finally, the instability of rock slope is predicted.

Most of the existing rock rheological model theories are the combination of linear elements or the combination of linear and nonlinear elements, which cannot describe the characteristics of accelerated creep stage well. With the development of the theory of damage fracture mechanics and the rheological theory of rock, it is a trend to establish the rheological model of rock with damage or fracture factors.

In this paper, the Caputo fractional derivative was used to establish a modified time-varying damage model.

Definition 1. The Caputo fractional derivative is defined as [13]

$$
{ }^{C} D_{0+}^{\alpha} f(t)=\frac{1}{\Gamma(n-\alpha)} \int_{0}^{t}(t-\tau)^{n-\alpha-1} f^{(n)}(\tau) d \tau, \quad t \geq 0, \epsilon,
$$

where the Gamma function is defined as $\Gamma(z)=\int_{0}^{\infty} t^{z-1} e^{-t} \mathrm{~d} t$.

By simulating the experimental data in [14], the nonlinear damage elements are considered in the acceleration stage. Through the parameter fitting software, we get the model parameters, which have high fitting degree and can fully reflect the acceleration stage.

\section{Viscoelastic Constitutive Model of Rock Slope with Damage}

The constitutive model of any kind of material must be able to fully express the internal structure, physical and mechanical properties of the material, and constitutive equation derived from the model can correctly reflect the properties of the material. Therefore, how to establish the creep constitutive model of rock slope becomes the key point in the study of rock slope creep stage. Only by establishing the correct constitutive model of rock slope creep, the relationship between stress, strain, and time and the creep characteristics of rock slope can be fully and accurately described [14].

The mechanical property of rock slope is not just elastic or viscous. To be more exact, it is a kind of viscoelasticity. Therefore, the study of rock slope constitutive relationship should not only be limited to the relationship between stress and strain but also need to consider the factor of time. If the classical elastic-plastic viewpoint is still used to analyze the mechanical properties of rock slope, there will be obvious defects, which will make it difficult to explain the phenomena of deformation with time in practice, and the long-term safety will also be threatened.

Here, we introduce three kinds of time-dependent mechanical elements as follows.

2.1. Time-Varying Elastic Element. In order to describe the attenuation stage of the creep curve, a time-varying elastic element is proposed. As shown in Figure 1, the elastic element coefficient of the model show an attenuation trend with the time $t$. The attenuation law is as follows:

$$
E(t)=\frac{E}{1-e^{-m t}}
$$

where $E$ is the initial value of the elastic element and $m$ is the dimensionless parameter determined by the simulation test.

2.2. Time-Varying Viscous Element. In order to characterize the viscosity variation of salt rock involving the sample degradation, an exponentially varying viscosity (see Figure 2) was proposed by Zhou et al. [15] and can be written as [16]

$$
\eta(t)=\eta_{0} e^{\phi t}
$$

where $\eta_{0}$ denotes the initial viscosity and $\phi$ is a constant.

2.3. Damage Analysis. Slope deformation prediction is one of the important topics in the research of slope stability, and it is also a worldwide scientific problem. The engineering geological conditions and the mechanism of slope deformation and failure are very complex, and the interaction effect and time effect of the basic factors cannot be described quantitatively. Therefore, it is difficult to master the law of slope deformation and failure and predict it. Aiming at the problem of dynamic slope stability which is more and more prominent in mine production, quantitative scientific monitoring means and forecasting methods are urgently needed to meet the orderly progress of mine safety production.

At present, there are few research studies on the creep of rock slope. However, there are numerous precedents of delayed construction and even failure due to insufficient 
research on rock properties including rheological properties of rock slope, such as Vajont bank creep failure in Italy.

The slopes of open-pit coal mines are mostly composed of sedimentary rocks. Some intercalations containing clayey materials are in the plastic state with obvious rheological characteristics due to the softening effect of groundwater, and their stress-strain relations are time-dependent. Almost all of the potentially unstable slopes are controlled by this clayey intercalation. When the stress state of the slope is less than a certain stress level, the slope will only be deformed; and when the stress level is greater than a certain stress level, the steady creep will occur. As time goes on, the steady creep will develop to accelerated creep, which will lead to the failure of the slope. If the stress level is high, the acceleration period will come sooner.

When the slope rock mass enters the accelerated creep stage, the internal damage of the rock mass begins to occur, and the relationship of the nonlinear accelerated damage with the increase of time is presented. As shown in Figure 3, the damage element is introduced to describe the state of accelerated creep of the rock mass, which is defined as [17]

$$
D=1-(\lambda \sigma)^{-\xi(t-\widehat{t})}
$$

where $D$ is the damage element, $\lambda$ and $\xi$ are constants, $\widehat{t}$ is the conversion time from the steady-state creep to the accelerated creep, and $\sigma$ is the stress.

\subsection{Modified Caputo Fractional Time-Varying Damage} Model. According to the actual situation of slope deformation and its geological environment factors, the above three mechanical elements are combined in series, as shown in Figure 4. Using the genetic effects of fractional derivatives, the modified Caputo fractional time-varying damage model is constructed, which describes the mechanical properties of damage. The stress of each element is equal to the total stress of the model, and the sum of the strains of each element model is equal to the total strain of the model. These two points are the basic basis for the establishment of the composite constitutive model.

Its corresponding equation of state is shown as

$$
\left\{\begin{array}{l}
\sigma_{1}=E(t) \cdot \varepsilon_{1}, \\
\sigma_{2}=\eta(t) \cdot{ }^{C} D_{0+}^{\alpha} \varepsilon_{2}, \\
\varepsilon_{3}=\frac{\sigma_{3}}{E_{3}(1-D)}, \\
\sigma=\sigma_{1}=\sigma_{2}=\sigma_{3}, \\
\varepsilon=\varepsilon_{1}+\varepsilon_{2}+\varepsilon_{3} .
\end{array}\right.
$$

Using Laplace transform, we have

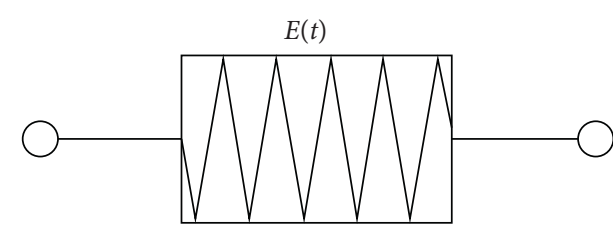

FIGURE 1: Time-varying elastic element.

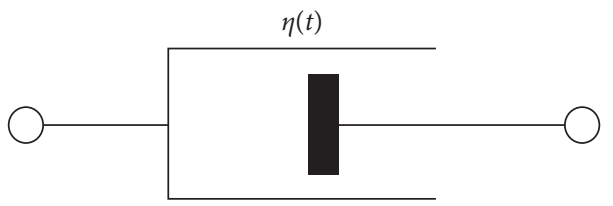

FIgURE 2: Time-varying viscous element.

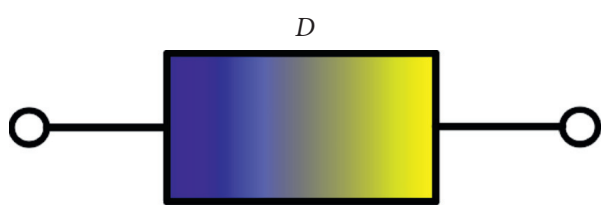

FIgURE 3: Damage element.

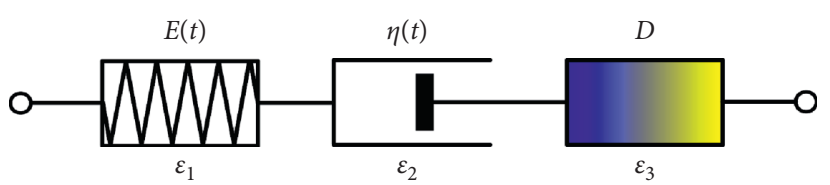

FIGURE 4: Modified Caputo fractional time-varying damage model.

$$
\varepsilon(t)=\frac{\sigma}{E_{1}}\left(1-e^{-m t}\right)+\frac{\sigma}{\eta_{0}} \cdot t^{\alpha} \cdot E_{1,1+\alpha}(\phi t)+\frac{\sigma}{E_{3}}(\lambda \sigma)^{\xi(t-\widehat{t})}
$$

where the Wiman function $E_{\chi,\rangle}(t)$ is [18]

$$
E_{\chi},(t)=\sum_{i=0}^{\infty} \frac{t^{i}}{\Gamma(i \chi+)}
$$

\section{Experimental Data Identification and Verification}

The deformation and fracture of rocks are complex processes with energy evolution between rocks and the external environment. The monitoring and prediction of the impending progressive failure is of great significance to ensure the stability of the rock structures and the safety of the workers [19].

The experimental data of creep curves in [14] show three typical deformation stages as attenuation, steady, 


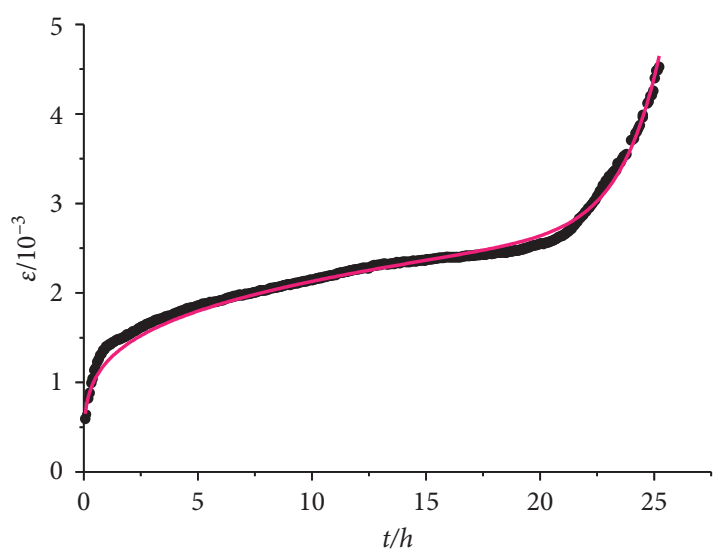

- Fitting curve, $R^{2}=0.9918$

- Experimental data

Figure 5: Fitting curve.

TABLE 1: Identified results of parameters.

\begin{tabular}{lccccccc}
\hline$E_{1}(\mathrm{MPa})$ & $E_{3}(\mathrm{MPa})$ & $\eta_{0}$ & $\phi$ & $\lambda$ & $\xi$ & $\alpha$ & $m$ \\
\hline 11370 & 18705.154 & 0.097 & 0.002 & $5.95 \times 10^{-6}$ & -0.072 & 0.766 & 2.567 \\
\hline
\end{tabular}

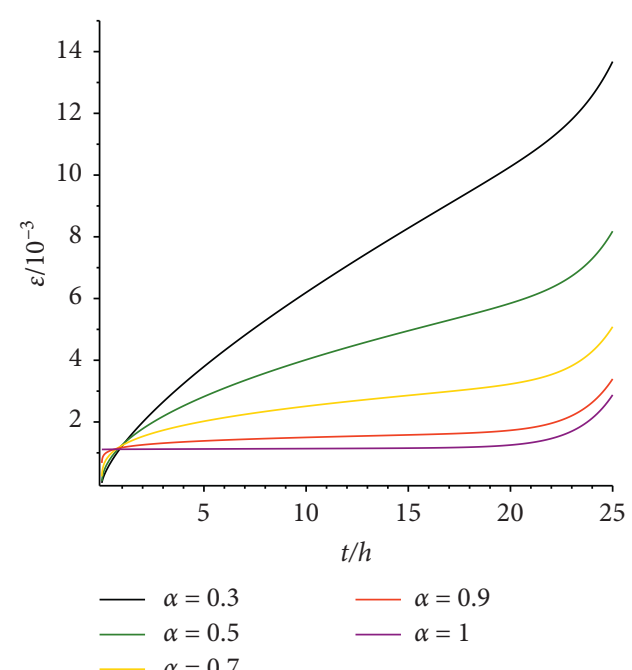

(a)

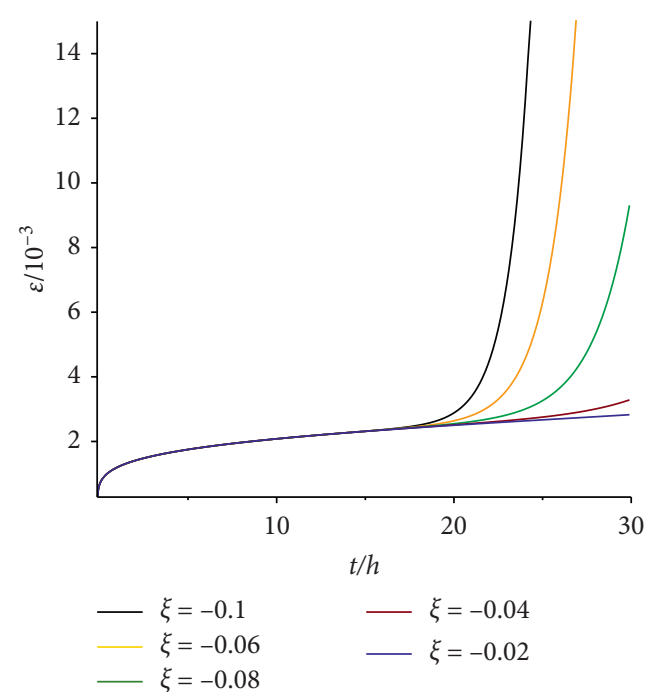

(b)

Figure 6: The creep curve of salt rock under the thermal effect.

and accelerated creep. Using the numerical simulation software, Figure 5 gives the creep experimental data and compares with the fitting curve of the modified Caputo fractional time varying damage model proposed in this paper. The fitting curve is in good agreement with the experimental data, which proves that the damage model proposed in this paper is reasonable and accurate. The parameters involved in the model are identified, and the results are shown in Table 1 .

\section{Model Prediction Analysis}

Using the fitting results in Table 1, as shown in Figure 6, we show the influence of the parameters of time-varying viscus element and damage element, $\alpha$ and $\xi$, on strain, respectively.

As $\alpha$ increases, the strain rates of the first and second stages of creep, attenuation and steady-state stages, gradually decrease. When $\alpha=1$, the attenuation creep stage disappears, 
which cannot fully reflect the whole rock creep situation. It indicates the importance of the fractional derivative. The strain rate in the acceleration stage is reflected in $\xi$, with the decrease of $\xi$, rock slope will enter the accelerated creep stage with high strain rate in advance. By adjusting the parameters, the failure of rock slope can be well predicted.

\section{Conclusions and Discussion}

In addition to the basic characteristics described by classical elastic-plastic mechanics, the stability and stress-strain state of slope also have a very obvious law that changes with time. On the one hand, the stress-strain state depends on the loading history; on the other hand, it changes with time, which determines the long-term stability of the slope.

The modified Caputo fractional time-varying damage model proposed in this paper reduces the number of model elements by using the series of time-dependent mechanical elements. Compared with other multielement models, the modified model has more concise constitutive equations, and it can better reflect the viscoelastic creep characteristics before the stress threshold and the viscoelastic plastic creep characteristics after the stress threshold with a certain reference value. Based on the creep test results, the parameters of the creep constitutive model of slope are determined by using the powerful nonlinear fitting function of mathematical software. The results truly reflect the damage of slope rock mass in the accelerated creep stage and provide a theoretical basis for the prediction of slope failure process.

\section{Data Availability}

Some or all data, models, or code generated or used during the study are available from the corresponding author by request.

\section{Conflicts of Interest}

The authors declare that they have no conflicts of interest.

\section{Acknowledgments}

This research project was made possible through the financial support from the National Key Research and Development Program of China under Grant no. 2017YFC0804600.

\section{References}

[1] T.-Q. Yang, Theory and Application of Viscoelasticity, Science Press, Beijing, China, 2004.

[2] W. Wang-Yi, Fluid Mechanics, Peking University Press, Beijing, China, 2013.

[3] W.-H. Tan, Theory and Practice of Generalized Reliability in Slope Engineering, Science Press, Beijing, China, 2010.

[4] B. Gordan, D. Jahed Armaghani, M. Hajihassani, and M. Monjezi, "Prediction of seismic slope stability through combination of particle swarm optimization and neural network," Engineering With Computers, vol. 32, no. 1, pp. 85-97, 2016.
[5] S. A. Elsoufiev, "Ultimate state of a slope at non-linear unsteady creep and damage," Slope Stability Engineering, vol. 1 \& 2, pp. 213-217, 1999.

[6] H. Liu, L. Li, S. Li, and W. Yang, "The time-dependent failure mechanism of rocks and associated application in slope engineering: an explanation based on numerical investigation," Mathematical Problems in Engineering, vol. 2020, Article ID 1680265, 19 pages, 2020.

[7] P. Liu, Y. Chen, Z. Yu, and Z. Lu, "Damage constitutive model and mechanical performance deterioration of concrete under sulfate environment," Mathematical Problems in Engineering, vol. 2020, Article ID 3526590, 16 pages, 2020.

[8] H. Zhang, H. Zhao, X. Zhang et al., "Creep characteristics and model of key unit rock in slope potential slip surface," International Journal of Geomechanics, vol. 19, no. 8, Article ID 04019094, 2019.

[9] F. Riva, F. Agliardi, D. Amitrano, and G. B. Crosta, "Damagebased time-dependent modeling of paraglacial to postglacial progressive failure of large rock slopes," Journal of Geophysical Research: Earth Surface, vol. 123, no. 1, pp. 124-141, 2018.

[10] P. Lacroix and D. Amitrano, "Long-term dynamics of rockslides and damage propagation inferred from mechanical modeling," Journal of Geophysical Research: Earth Surface, vol. 118, no. 4, pp. 2292-2307, 2013.

[11] L. Xue, S. Qin, P. Li, G. Li, I. Adewuyi Oyediran, and X. Pan, "New quantitative displacement criteria for slope deformation process: from the onset of the accelerating creep to brittle rupture and final failure," Engineering Geology, vol. 182, pp. 79-87, 2014.

[12] R. K. Umrao, R. Singh, L. K. Sharma, and T. N. Singh, "Soil slope instability along a strategic road corridor in Meghalaya, north-eastern India," Arabian Journal of Geosciences, vol. 10, no. 12, p. 260, 2017.

[13] M. Caputo, "Linear models of dissipation whose Q is almost frequency independent-II," Geophysical Journal International, vol. 13, no. 5, pp. 529-539, 1967.

[14] C. Y. Lu, "Study on the creep and stability drop prediction of rock slope," Master's Thesis, Xiang Tan University, Xiangtan, China, 2016.

[15] H. W. Zhou, C. P. Wang, L. Mishnaevsky, Z. Q. Duan, and J. Y. Ding, "A fractional derivative approach to full creep regions in salt rock," Mechanics of Time-dependent Materials, vol. 17, no. 3, pp. 413-425, 2013.

[16] X. Yang, W. Cai, Y. Liang, and S. Holm, "A novel representation of time-varying viscosity with power-law and comparative study," International Journal of Non-linear Mechanics, vol. 119, Article ID 103372, 2020.

[17] Y.-z. Jiang, "Nonlinear creep damage constitutive model of rock," Journal of China University of Mining \& Technology, vol. 38, no. 3, 2009.

[18] A. Wiman, "Über die Nullstellen der Funktionen Ea(x)," Acta Mathematica, vol. 29, pp. 217-234, 1905.

[19] Q. Guo, J. Pan, M. Cai, and Y. Zhang, "Analysis of progressive failure mechanism of rock slope with locked section based on energy theory," Energies, vol. 13, no. 5, p. 1128, 2020. 